\title{
Pobreza, consumo y defensa del consumidor
}

Ismael Núñez Sáenz

\section{Introducción}

En la actualidad, el Derecho debe afrontar dos grandes problemas frente al reto de ser un instrumento de cambio social en una realidad cambiante.

El primero de ellos es la Estática (relativa) -entendida como resistencia al cambio- que presenta y le es propia, frente a la dinámica social, ajena a "formalismos" y que no espera los cambios que el Derecho pueda sufrir en su intento de acompañarla.

El segundo problema es el "repliegue forzado" a que es sometido por teorías económicas (nada novedosas) que pretenden que sea el mercado quien solucione cuanto problema social exista.

El estatismo del Derecho puede encontrar solución si hallamos la forma de inyectarle dinámica; obviamente ella no puede fundamentarse en la simple producción y sobreproducción normativa, en un "dinamismo formal" que en si es un concepto contradictorio, creemos que en todo caso el Derecho debe encontrar esta dinámica en la renovada lectura e interpretación de sus normas.

La interpretación de una norma desde la realidad, permite ampliar los horizontes y alcances de la misma. Un enfoque diferente de un mismo problema, puede generar a partir de una norma pre-existente ya no una sino dos, tres o más soluciones, sin necesidad de modificarla.

Por otro lado el "repliegue forzado" del Derecho, fundamentado en el liberalismo económico es más una cuestión principista. Ciertamente, no se puede cerrar los ojos ante una realidad que básicamente se mueve por las fuerzas económicas de la oferta y la demanda, ni dejar de 
percibir las consecuencias que ello traerá a la postre -sino desde ya- al Derecho.

Sin embargo también es cierto que en tanto los mercados sean incapaces de funcionar adecuadamente, no podrán aportar soluciones apropiadas a los problemas que se presenten, y en esa medida el Derecho tendrá aún mucho que hacer.

La defensa del consumidor y la problemática de los productos denominados "basura" pueden ser leídas desde cualquiera de las dificultades que afronta el Derecho de nuestros días. No sólo existe una "retirada" del Derecho ante la realidad del consumo en un país pobre, sino que existe a la par el reto constante de recrear -desde la defensa del consumidor- una solución adecuada.

El presente ensayo aborda esta temática desde ambos vértices, convencidos que no es el mercado el llamado a dar "las soluciones" por estar inhabilitado para ello (como se verá) sino que es el Derecho el responsable de hacerlo. Esta tarea será cumplida eficazmente siempre y cuando la defensa del consumidor sea enfocada, no solamente desde el punto de vista económico-liberal que ve en nuestra economía un mercado que funciona a la perfección, sino además desde un punto de vista sociológico, que ve en la pobreza una problemática pre-existente, que cuestiona constantemente los fundamentos del mercado.

Por ello pobreza, consumo y defensa del consumidor serán los puntos de partida de un análisis que pretende aportar al derecho en la medida que éste decida responder a nuestra realidad.

\section{Teoría y realidad}

\subsection{El consumo basura: una constatación fáctica ${ }^{1}$.}

No es necesario enumerar la cantidad de productos de baja y pésima calidad que nuestra economía produce, en los hechos nadie es ajeno al

1 El consumo basura supone la existencia de "productos basura". A lo largo del presente ensayo, al referirnos a productos basura, aludiremos a aquellos productos de baja y pésima calidad siempre que no vaya asociada a un signo distintivo añadido al producto, de manera ilegítima (producto bamba). La distinción es poco técnica pero válida, los productos bamba atentan contra derechos de propiedad (signos distintivos), los productos basura no, lo que nos exime de analizar éstos desde un punto de vista real. Si no hiciésemos la diferencia, tendría- 
consumo de algún tipo de producto basura, sin embargo es significativo que en los sectores más deprimidos de la población, este tipo de bienes inferiores se conviertan en bienes normales ${ }^{2}$. Obviamente es insoportable que existan personas que consuman carne descompuesta sin saberlo, o que coman cartón en vez de hamburguesas.

No obstante ello, más que un problema ético que podamos solucionar con algún imperativo categórico, responde a una realidad económica clara: la pobreza de nuestros consumidores.

Es para esta realidad pobre, que se han esgrimido algunos argumentos a saber, que pretenden explicar y justificar al final, la existencia de productos de pésima calidad.

\section{2. ¿Cómo se explica el consumo de productos basura? ${ }^{3}$}

En primer lugar, se argumenta que nuestra economía es de libre mercado y que para ser consistente con ella, el Estado debe abstenerse de controlarla y dejar que sea la "mano invisible" de la economía, quien corrija las imperfecciones del mercado.

Por otra parte, reforzando este argumento, se dice que la existencia de los "productos basura" y sus consecuencias (léase externalidades) pueden ser internalizadas por los consumidores ya que finalmente ellos son "racionales" en sus decisiones de consumo al realizar un análisis costo-beneficio en cada decisión. Los consumidores pobres no serían la excepción al supuesto de racionalidad pues ellos, sobre todo, maximizan la utilidad de cada sol que ganan.

Finalmente se afirma que si el Estado interviniese el mercado de estos productos para "controlar calidad" se generarían externalidades (sobrecostos) que difícilmente podría asumir el consumidor pobre, al

mos que incorporar este análisis excediendo por seguro el límite de extensión permitido para este tipo de trabajos. Ello nos alivia por ahora, pero no nos releva de abordar en un futuro la problemática de los productos bamba.

2 Un bien normal es aquel cuya cantidad demandada a cada uno de los precios aumenta cuando aumenta la renta. Un bien inferior es aquel cuya cantidad demandada disminuye cuando aumenta la renta.

3 Aquí exponemos en forma resumida lo que creemos son los argumentos centrales de la propuesta liberal en estos temas, expuestos por el Dr. Alfredo Bullard González en su artículo " ;lo que no mata engorda; , los productos basura y los perjuicios y prejuicios de la protección al consumidor en un país pobre"; aparecido en la revista Ius et Veritas No 12 Lima, 1996 pp. 103-113. 
que precisamente queremos proteger con dicho control. En síntesis el efecto sería contraproducente.

En qué medida estos argumentos son válidos en una realidad pobre como la peruana es lo que a continuación analizaremos.

\section{Consumo: una visión desde la pobreza.}

Creemos que toda la argumentación que trata de explicar la existencia y futura persistencia de los "productos basura" tiene como punto de partida la idea de un mercado que cumple con los presupuestos básicos para viabilizar una economía absolutamente liberal, sin embargo ello no es así.

Nuestra tesis (a demostrar) es que en el sector de pobreza, pero sobre todo de pobreza extrema de nuestra economía, más de un presupuesto del mercado no se cumple; si ello es así no podría liberalizarse sin más, la producción de bienes y servicios en este sector de la economía, por el contrario, habría que corregir las imperfecciones en los presupuestos del mercado y ello implica como sabemos, intervención directa o indirecta del Estado pues es él quien plantea el marco normativo e institucional, "las reglas de juego básicas" para el desarrollo de una economía libre .

\subsection{La pobreza en el Perú5.}

Antes de analizar las implicancias económicas y legales de la pobreza a propósito de la defensa del consumidor es necesario establecer algunas cuestiones previas. En primer lugar, ¿quiénes pueden ser considerados

4 Como sostiene Douglass North. En Preguntas a Douglass North. Documento de trabajo basado en extractos de libro: Instituciones, Cambio Institucional, y Desempeño Económico. Editado por el Fondo de Cultura Económica. Preparado para la visita del profesor Douglass North al Perú. Lima, Junio de 1995.

5 Los datos que se mencionarán a continuación se han obtenido de la Encuesta Nacional sobre Medición de Niveles de Vida (ENNIV 94) que se realizará entre mayo y setiembre de 1994. La ENNIV 94 fue ejecutada por Cuánto S.A. con el auspicio del Banco Mundial y el Banco Interamericano de Desarrollo. Los primeros resultados de la encuesta aparecieron en la Revista Cuánto, actualmente se cuenta con una publicación hecha por el Instituto Cuánto y UNICEF bajo el título: Retrato de la Familia Peruana, niveles de vida, 1994. 2 tomos. Lima. Editorial Navarrete, 1996. 
pobres? Para la ENNIV 94 son pobres las personas cuyo gasto total no cubre el costo de una canasta básica de consumo de alimentos y otros bienes y servicios. Ahora bien, dentro de la categoría de pobres existe otra, de pobres extremos, referida a aquellas personas cuya alimentación no satisface ni siquiera los requerimientos mínimos nutricionales ${ }^{6}$.

En segundo lugar, ¿Cuántos son los pobres en el Perú? Aunque parezca increíble, la mitad de la población peruana es decir 11.5 millones de personas aproximadamente, se encuentran viviendo en situación de pobreza. Según los cálculos realizados para el año 1994 la proporción de pobres equivale al $49.6 \%$ de la población total del país. Asimismo, se estimó que la población que vive en pobreza extrema representa alrededor de 4,7 millones, lo que quiere decir que 1 de cada 5 personas, padece de hambre en el Perú ${ }^{7}$.

Revisadas estas dramáticas estadísticas queda analizar en qué medida esta situación de pobreza cuestiona ciertos presupuestos del mercado. Como sabemos son presupuestos básicos: la existencia de individuos racionales; la inexistencia de externalidades generadas por la contratación; información de mercado suficiente; inexistencia de monopolios u oligopolios y un sistema contractual viable en términos de costos.

La problemática de la pobreza, cuestiona directamente los dos primeros presupuestos: La racionalidad del consumidor y la necesaria ausencia de externalidades contractuales. Un tercer presupuesto en cuestión y a analizar es la información en el mercado de productos basura, pero cabe resaltar que si bien la escasez de información tiene alguna incidencia sobre la pobreza de nuestros consumidores, en última instancia trasciende su problemática.

\subsection{La racionalidad del consumidor}

¿Qué consumidor es más racional, aquel náufrago que decide comer su zapato por hambre; el mendigo que come basura por idénticas razones o el consumidor que decide no comprar más un producto y dirigir su preferencia hacia otro, porque el primero no le satisface?

Intuitivamente podríamos afirmar que todos son racionales en la medida que realizan comportamientos maximizadores y optan por la

6 Retrato de la Familia Peruana, niveles de vida, 1994, Tomo I p. 28.

7 Ibid p. 29. 
alternativa que les produce un mayor beneficio ${ }^{8}$. Sin embargo, sabemos que tanto el náufrago como el mendigo se mueven en un mundo de "dos" alternativas: morir de hambre o comer zapatos o basura respectivamente por lo que podríamos afirmar válidamente que en realidad no tienen alternativas. Más, si todos los personajes de nuestro ejemplo son sujetos económicos maximizadores capaces de decidir lo mejor para cada uno no se entiende bien, por que la propuesta de liberalizar el mercado de "productos basura" encuentra resistencia, pues ¿̨finalmente no es el consumidor quien mejor decide, pues conoce sus preferencias y presupuesto? ¿Por qué entonces verlo como un "tonto" incapaz de decidir??

Creemos que el problema de la propuesta liberal y de la aparente consistencia de sus argumentos reside en esto último, en asimilar el concepto de "consumidor racional" al de "individuo racional" 10 , inteligente, con capacidad de discernimiento y oponerlo al de "consumidor tonto".

En principio el símil es correcto pero no absoluto, en virtud de ello es necesario afinar el concepto de "consumidor racional" y llevarlo un poco más allá de la concepción de individuo racional (sujeto económico) que si bien le sirve de base no gráfica su real complejidad. La noción de consumidor racional es más técnica que la de individuo racional. Para la teoría económica, consumidor racional será un tipo específico de individuo racional; aquel que pueda maximizar su utilidad en función de una canasta elegida (con lo que se llega al óptimo del consumidor), elección que supone a su vez, la existencia de un conjunto ordenado de preferencias ${ }^{11}$.

Hace tiempo la autorizada opinión de Gary Becker sostuvo que cuando no existe opción, no existen tampoco problemas de elección ${ }^{12}$. Esto es precisamente lo que sucede en la economía de gran parte de

8 De acuerdo a la noción que el Análisis Económico del Derecho maneja de "Conducta racional". Torres López, Análisis económico del Derecho, Madrid, Tecnos S.A. 1987. pp. 30-31.

9 Alfredo Bullard G. " $i$ Lo que no mata engorda" op. cit. p. 105.

10 Que en sí es un presupuesto del Análisis Económico. Torres López, op. cit. p. 31.

11 Gary S. Becker. Teoría Económica, México, Fondo de Cultura Económica, 1977 p. 41; Amalia Cuba Salerno, Pobreza Urbana; una visión desde el consumo. Lima, FOMCIENCIAS, 1991 p. 9; C. Ferguson, Teoría Microeconómica. México, Fondo de Cultura Económica. 1973.

12 Gary S. Becker. Op. cit. p. 11. 
nuestros consumidores: su situación de pobreza y niveles bajísimos de ingreso los condenan en la práctica a aceptar lo que sea para subsistir.

Le ENNIV 94 revela que del total de hogares del país, el 10\% tiene un nivel de ingresos promedio, 13 veces superior al obtenido por el $10 \%$ de hogares más pobres, además se comprobó que el ingreso promedio de los hogares de las ciudades es aproximadamente el doble del obtenido por los hogares del campo ${ }^{13}$. Por otra parte, tanto en Lima Metropolitana como en las demás capitales de departamento, desde abril de 1994 a agosto de 1996, la remuneración mínima vital mensual se mantuvo tan sólo en $\mathrm{S} / .132 .00^{14}$, y aunque es cierto que en los hogares del Perú los ingresos provenientes del trabajo dependiente representan el $27.5 \%$ del total de sus fuentes ${ }^{15}$, ello no quiere decir que la situación de los hogares pobres y pobres extremos (dedicados básicamente a la actividad independiente) sea mejor. Ello se puede apreciar en los siguientes gráficos.

\section{GASTO PROMEDIO PER CÁPITA MENSUAL POR HOGAR SEGÚN ÁREAS GEOGRÁFICA 1994}

(Nuevos Soles de diciembre de 1994 a precios de Lima Metropolitana)

\begin{tabular}{lcccc} 
& $\begin{array}{c}\text { Total de } \\
\text { la población }\end{array}$ & Extremos & Total & No pobres \\
\cline { 3 - 4 } & & & & \\
Promedio Nacional & 183 & 53 & 86 & 277 \\
Lima Metropolitana & 241 & 62 & 111 & 323 \\
Costa Urbana $\left(^{*}\right)$ & 204 & 61 & 99 & 302 \\
Costa Rural & 133 & 56 & 81 & 235 \\
Sierra Urbana & 203 & 56 & 95 & 280 \\
Sierra Rural & 107 & 52 & 66 & 193 \\
Selva Urbana & 152 & 45 & 65 & 204 \\
Selva Rural & 73 & 39 & 50 & 129
\end{tabular}

(*) No incluye Lima Metropolitana

Nota: Los valores monetarios de junio de 1994 obtenidos en la ENNIV han sido ajustados con la inflación a precios de diciembre de 1994

Fuente: Cuánto S.A. ENNIV (En Cuánto: Enero 1995, p. 6)

13 "Encuesta. Hogares Peruanos: Examen de Ingreso". En Revista Cuánto. Lima, julioagosto 1996 , p. 4.

14 Perú: Compendio Estadístico 1995-1996. Lima INEI. Agosto 1996 pp. 379-380.

15 Otras fuentes son: El trabajo independiente (39.5\%); el capital (10.1\%); las transferencias $(7.4 \%)$; el autoconsumo ( $11.8 \%)$ y otros $(2.7 \%)$. "¿De dónde vienen los ingresos? Principales fuentes de ingreso del hogar, según dominios de estudio" 1994, en: Encuesta Hogares Peruanos: Examen de ingreso. Op. Cit. p. 7. 


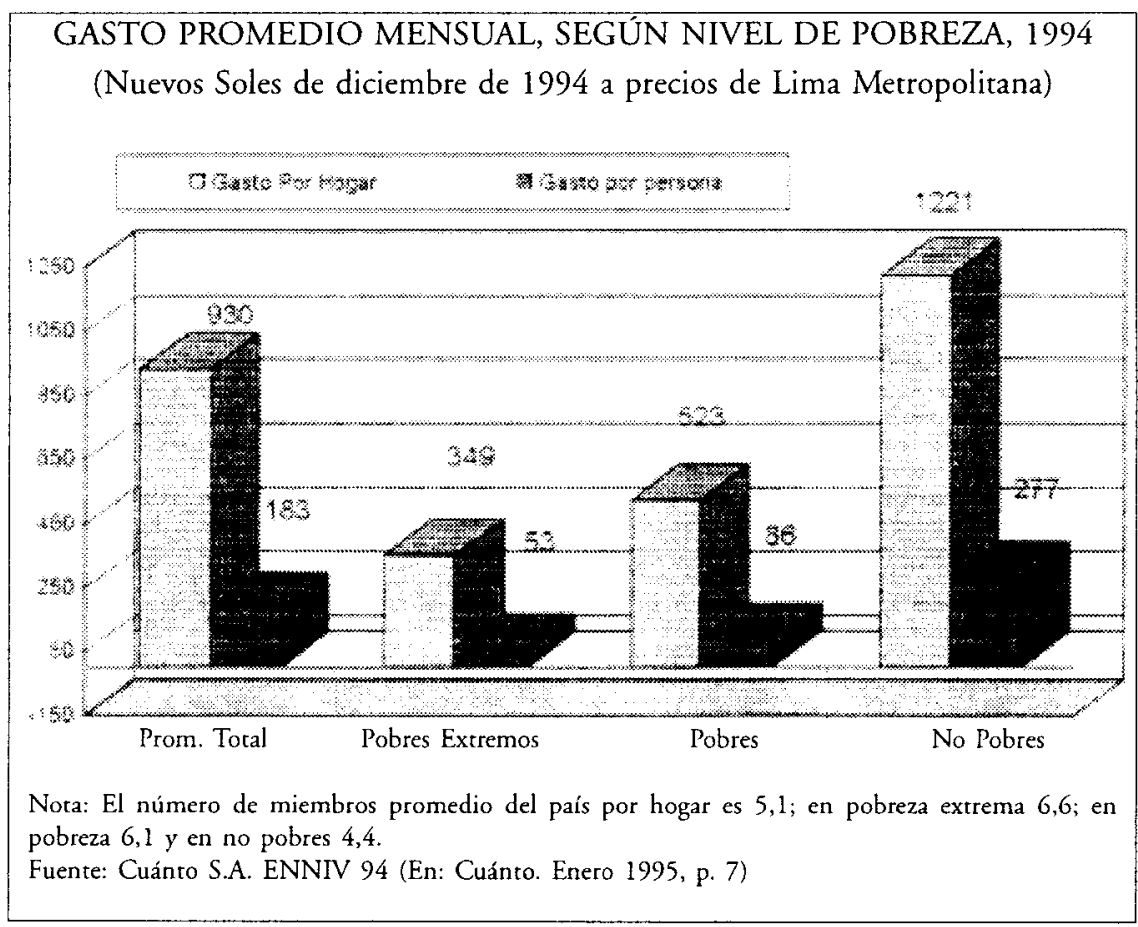

Como se puede apreciar, el gasto de un pobre extremo, es decir de aquellos cuyo gasto total no llega a cubrir el costo de una canasta básica alimentaria, representa un quinto del gasto de alguien que se encuentra por encima de la línea de pobreza. Ello explica por qué gran proporción del gasto de los hogares de bajos ingresos se destina a la alimentación y satisfacción de otras necesidades básicas, lo que los deja con un escaso margen para maximizar utilidad mediante la sustitución de bienes frente a diferentes conjuntos de precios. ${ }^{16}$

Existen pues "niveles de consumo críticos" o de "infraconsumo" en las familias pobres del Perú, niveles que sólo una vez superados permiten reemplazar el consumo de un bien por otro de calidad superior ${ }^{17}$.

16 Esto es lo que Amalia Cuba a denominado "gasto rígido" que lo identifica con el conjunto de demandas asociadas a un bloque, del cual no puede sustraerse el consumidor aun cuando sea pobre y que significan más de las $2 / 3$ partes de su gasto total. Amalia Cuba Salerno, Op. cit. p. 61.

17 Ibid. P. 9. 
DISTRIBUCION DEL GASTO POR GRUPOS DE CONSUMO 1994 (Porcentaje)

\begin{tabular}{lrrrr} 
& \multicolumn{4}{c}{ Pobres } \\
\cline { 2 - 5 } & Total & Extremos & Total & No Pobres \\
& 100.0 & 100.0 & 100.0 & 100.0 \\
Total & 49.4 & 68.1 & 61.2 & 45.8 \\
Alim. y Beb. $\left(^{*}\right)$ & 6.0 & 7.0 & 6.1 & 5.9 \\
Vest. y Calz. & 24.2 & 15.1 & 19.8 & 25.6 \\
Alq. Comb. y Electricidad & 3.0 & 3.4 & 2.9 & 3.0 \\
Muebles y Enseres & 1.1 & 0.4 & 0.7 & 1.2 \\
Cuid. de Salud & 6.3 & 2.5 & 4.2 & 7.0 \\
Transp. y Comun. & 6.2 & 2.6 & 3.6 & 6.9 \\
Esparc. y Cultura & 1.3 & 0.3 & 0.5 & 1.6 \\
Otros bienes y Servicios & 2.5 & 0.5 & 0.8 & 3.0 \\
Transferencias & & & & \\
& (*) Incluye alimentos y bebidas fuera del hogar. \\
vente: Cuánto S.A. - ENNIV 94 (Estimados preliminares) & & \\
n: Cuánto. Enero 95 p. 9.
\end{tabular}

Sin embargo la realidad puede ser y de hecho es mucho más dramática. Si revisamos la distribución porcentual de necesidades básicas satisfechas, insatisfechas y en miseria de algunos departamentos del Perú ${ }^{18}$ (v. gr. Huancavelica, Cajamarca, Cuzco e incluso Lima) comprobaremos que la gran mayoría de la población, que se encuentra en situación de pobreza o pobreza extrema; y que destina una gran parte de sus ingresos a satisfacer necesidades básicas, mantienen incluso altos índices de insatisfacción de las mismas.

Esta constante pérdida de la capacidad adquisitiva ${ }^{19}$ de las familias pobres del Perú, en la práctica tornan inoperante la racionalidad del consumidor en sus decisiones de compra, pues su posibilidad de elección es mínima y si no se puede elegir, ¿¿de qué "consumidor racional" hablamos? Ello no quiere decir que el consumidor pobre sea incapaz de decidir qué es lo que le conviene o lo que no le conviene (por ejemplo los "productos basura") sino tan solo que su deteriorada economía no le permite "comparar" y por ende deducir "qué es lo mejor". Está obligado a aceptar lo que el mercado produzca a determinado nivel de

18 Perú: Compendio Estadístico $1995-1996$ p. 163.

19 "En la última década según la ENNIV 94, los niveles del gasto real de los peruanos se han deteriorado en un 64\%. Si en julio de 1985 una persona gastaba en alimentación, vivienda, vestido y otros bienes y servicios 100.00 nuevos soles, actualmente sólo gasta 36.00 nuevos soles". "El Gasto de Cada Peruano". En Revista Cuánto, Lima, enero 1995. p. 5. 
PERÚ: DISTRIBUCIÓN PORCENTUAL DE LA POBLACIÓN CON

NECESIDADES BÁSICAS SATISFECHAS, INSATISFECHAS Y EN MISERIA E INDICADORES, SEGÚN DEPARTAMENTO

(Ordenada en función al porcentaje de población con NBI) - (Censo Nacional de 1993)

\begin{tabular}{|c|c|c|c|c|}
\hline \multirow{3}{*}{ Departamento } & \multirow{3}{*}{$\begin{array}{c}\text { Población } \\
\text { En } \\
\text { Estudio }\end{array}$} & \multicolumn{3}{|c|}{ Población (\%) } \\
\hline & & \multicolumn{2}{|c|}{ Con Necesidades Básicas } & \multirow{2}{*}{$\begin{array}{c}\text { En } \\
M \text { iseria }\end{array}$} \\
\hline & & Sarisfechas & Insatisfechas & \\
\hline Total & 21419238 & 43.2 & 56.8 & 28.3 \\
\hline Huancavelica & 382436 & 4.8 & 92.2 & 51.0 \\
\hline Ayacucho & 485417 & 16.7 & 83.3 & 50.8 \\
\hline Apurímac & 378038 & 16.8 & 83.2 & 47.9 \\
\hline Pasco & 223259 & 18.8 & 81.2 & 44.6 \\
\hline Cajamarca & 1254056 & 20.9 & $\overline{79.1}$ & 51.7 \\
\hline Huánuco & 649430 & 21.0 & 79.0 & 47.8 \\
\hline Loreto & 674013 & 21.3 & 78.7 & 52.8 \\
\hline Amazonas & 333970 & 22.0 & 78.0 & 51.5 \\
\hline Madre de Dios & 64916 & 23.2 & 76.8 & 45.4 \\
\hline Cuzco & 1019579 & 24.1 & 75.9 & 40.6 \\
\hline Puno & 1071330 & 26.5 & $73 . \overline{5}$ & 33.7 \\
\hline U cayali & 308163 & 26.7 & 73.3 & 43.9 \\
\hline San Martín & 542780 & 28.1 & 71.9 & 46.8 \\
\hline Piura & 1377572 & 29.7 & 70.3 & 41.1 \\
\hline Junin & 1022642 & 33.9 & 66.1 & 29.4 \\
\hline Ancash & 948542 & 35.9 & 64.1 & 29.9 \\
\hline Tumbes & 149010 & 38.4 & 61.6 & 30.8 \\
\hline La Libertad & 1259681 & 49.4 & 50.6 & 22.1 \\
\hline Lambayeque & 914028 & 51.4 & 48.6 & 19.0 \\
\hline Moqueguia & 124080 & 52.1 & 47.9 & 20.3 \\
\hline $\mathrm{Ica}$ & 559687 & 55.7 & 44.3 & 15.3 \\
\hline Arequipa & 904884 & 56.7 & 43.3 & 18.6 \\
\hline Tacna & 212314 & 61.3 & 38.7 & 13.9 \\
\hline Lima & 6312672 & 65.5 & 34.5 & 11.9 \\
\hline Callao & 629175 & 66.6 & 33.4 & 11.4 \\
\hline
\end{tabular}

NBI: Necesidades Básicas Insatisfechas.

Fuente: Instituto Nacional de Estadística e Informática.

Dirección técnica de Demografía y Escudios Sociales. 
precios y, en todo caso, esta "incapacidad" de elección no proviene de su intelecto (estupidez) sino de su bolsillo.

En definitiva la existencia de consumidores sin capacidad de elección determina su irracionalidad en el plano del consumo y con consumidores irracionales el mercado no puede funcionar, razón ésta, por demás justificante de una intervención del Estado en el mercado, sino para corregir el origen de dicha irracionalidad, por lo menos para paliar sus consecuencias ${ }^{20}$.

\subsection{Las externalidades}

Que los contratos no generen externalidades, además de ser un presupuesto del mercado es uno de los argumentos por la no intervención estatal de la economía. Aquí queremos analizar ambos aspectos.

En primer lugar, la contratación de "productos basura" genera de hecho externalidades negativas, tal vez de ellas lo más preocupante sea los daños que determinados productos generan a la salud.

Una hamburguesa de cartón, un embutido elaborado en condiciones insalubres, un medicamento vencido, generan definitivamente efectos nocivos a la salud de los consumidores. Sin embargo, en la actualidad los precios de una gran cantidad de estos productos mantienen niveles que no reflejan en lo mínimo los costos que generan. Ello, lejos

20 Podríamos crear a partir de esta problemática una suerte de teoría de la Lesión Social. Como sabemos el artículo 1447 de nuestro código civil recoge la teoría de la lesión y la fundamenta en un aspecto subjetivo y otro objetivo. El subjetivo consta de dos elementos: el estado de necesidad en una de las partes y el aprovechamiento de la otra. Cuando el ordenamiento interviene en los contratos donde media lesión es porque entiende que una de las partes (la lesionada) no está en aptitud de garantizar un comportamiento maximizador que le brinde una utilidad particular y brinde finalmente una utilidad social. El estado pues, tiende a intervenir para promover la asignación eficiente de los recursos alli donde obviamente no se da. Similar situación encontramos en los sectores deprimidos de nuestra economía. El estado de necesidad del consumidor pobre, determinado por su ingreso y manifestado en sus niveles de gasto; actualmente es aprovechado por un conjunto de comerciantes inescrupulosos que no escatiman en ofrecer productos de pésima calidad, sin control alguno amparándose en la existencia de un "mercado libre".

Esta teoría (a desarrollar) de la Lesión Social podría justificar también, la ampliación de las fronteras de la defensa del consumidor, es decir la intervención del mercado, dado que es necesario promover la asignación eficiente de los recursos, en el sector de la economía en el cual el mercado no puede hacerlo por sí mismo. 
de ser una ventaja es una desventaja, pues la sociedad en general se perjudica con la asignación ineficiente de recursos, que por definición son escasos.

Los precios de gran parte de los "productos basura" (sobre todo los destinados a la alimentación) no indican nada sobre los graves efectos que generan en la salud de los pobladores, siempre ha merecido la atención del ordenamiento legal, se pretenda negar hoy en día dicha protección, so pretexto de no generar externalidades y sobrecostos en un mercado de productos de pésima calidad.

Si relacionamos salud y pobreza, comprobaremos que a menor cantidad de recursos las familias tienden a gastar menos en el cuidado de su salud y es que de acuerdo a estudios realizados ${ }^{21}$, en los hogares pobres, el gasto en salud es aleatorio ${ }^{22}$, de allí lo poco que las familias de bajos ingresos invierten en este concepto $(1.1 \%)^{23}$, lo que no hace sino confirmar el constante deterioro del nivel de vida de los peruanos.

Esta situación deviene más crítica para quienes se encuentran en pobreza extrema, los que dedican un preocupante $0.4 \%$ de su gasto a este rubro, lo que agrava más sus ya precarias condiciones de salud ${ }^{24}$.

Mantener en el mercado, sin ningún control, productos que generen un sin número de externalidades a la salud de los consumidores, más aún si éstos son pobres, es obligarlos a distraer una mayor cantidad del gasto en el cuidado de la salud, lo que va en detrimento del gasto que se realiza en alimentación y otras necesidades básicas.

De allí la necesaria participación del Estado y otras instituciones, en el mercado, en defensa del consumidor, para corregir esta sobreproducción de externalidades. Los métodos correctivos pueden ser diversos (como se verá en el siguiente capítulo) pero lo invariables es la intervención.

Por otro lado, la presencia del Estado en la economía, para controlar la producción o prohibir eventualmente la comercialización de de-

21 Amalia Cuba Salerno. Op. cit. p. 13.

22 En la medida que es asumido como "lucha contra la enfermedad" (no tiene naturaleza preventiva), por ello es un distorcionante de la estructura del gasto. Amalia Cuba Salerno, Op. cit. p. 61 .

23 "El gasto de cada peruano". Op. cit. p. 8.

24 Ibid. 
terminados "productos basura", va a crear externalidades. Sin embargo, ello no quiere decir que todas sean negativas. Por el contrario, creemos que dicho control generará a la postre externalidades positivos, pues los precios de estos productos estarán más acorde con los costos que generen, todo lo cual redunda no sólo en beneficio de la sociedad, que logra una asignación más eficiente de sus recursos sin mayor $\operatorname{costo}^{25}$, sino sobre todo del consumidor pobre, quien se verá beneficiado con un mínimo de calidad en los productos que aún se ve obligado a consumir.

Finalmente el control de calidad -se afirma- puede ser tan perjudicial como un control de precios, por ende habría que abstenerse de ello y aceptar que la calidad para los pobres es un bien suntuario ${ }^{26}$.

Una vez más estamos ante una verdad a medias. En definitiva la calidad cuesta y es natural que el incremento de los precios del producto sea directamente proporcional a la calidad que estos presenten.

A los proveedores de "productos basura", (sobre todo, los destinados a la alimentación o que puedan afectar la salud), no se les puede exigir que cumplan con los estándares de calidad ISO 9000 o ISO 9001; en cambio, si se les puede pedir que, dentro de los diversos niveles de calidad, cumplan con aquel nivel mínimo en el cual calidad sea sinónimo de seguridad (del producto y para el consumidor). Definitivamente la seguridad no puede ser un bien suntuario para el consumidor (sobre todo lo referido a la salud e integridad física), sino todo lo contrario, es un Derecho el cual, además, la sociedad siempre se ha preocupado en salvaguardar ${ }^{27}$.

\subsection{La información}

Referirse a la información como presupuesto del mercado no es en realidad asimilar esta idea a la de "información perfecta" sobre cada bien o servicio que se produce, es referirse si, a la información suficien-

25 Siempre se ha dicho que "no existe almuerzo gratis" pero si partimos del supuesto que la sociedad está produciendo ineficientemente, siempre es posible producir una cantidad mayor de todos los bienes y no es necesario perder nada produciendo algo más. En ese sentido existe algo "gratis". Stanley Fischer, Rudiger Dornbusch, Richard Schemalesse. Economía. Madrid. Mc. Graw-Hill. 2o edición en español 1995. p. 19.

26 Alfredo Bullard G. "iLo que no mata engorda,". Op. cit. p. 106.

27 Art. $7^{\circ}$ y Art. $65^{\circ}$. Constitución Política; Arts. 286-295\%; Código Penal; Art. $5^{\circ}$ inc. A; D. Leg. 716; por citar algunos ejemplos. 
te y relevante. Este tipo de información es necesaria, pues de ella depende en mucho la consistencia de la conducta racional. Alguien podría ser totalmente racional en sus decisiones, sin embargo, eventualmente podría decidir ineficientemente dada su falta de información, es decir ante dos alternativas, optar por aquella que no maximiza su utilidad ${ }^{28}$.

Como ya habíamos anunciado, la problemática de la información insuficiente del mercado de "productos basura" trasciende la problemática de la pobreza, es algo que afecta tanto a pobres como a no pobres, consumidores racionales como irracionales. Por ello, para demostrar que la falta de información justifica por si sola la imposibilidad de liberalizar el mercado de estos productos (a pesar de los argumentos en contra ya mencionados: la irracionalidad, el consumidor pobre y la generación de externalidades), partamos del supuesto que los consumidores pobres y pobres extremos, no obstante sus bajos ingresos y capacidad de elección casi nula, son racionales es decir poseen alternativas de consumo, aunque sean limitadas.

Ahora bien, ¿en qué medida los "productos basura" informan de manera suficiente sobre calidad, cualidades, precauciones, riesgos, etc. a los consumidores? La respuesta es sencilla, de ninguna manera.

El problema de información asimétrica que se genera es claro y ello debe ser corregido básicamente por tres razones: En primer lugar, por los riesgos que genera la desinformación. Actualmente el intercambio de "productos basura" se realiza en un mundo en el que la información prácticamente no existe, ello no permite aquilatar adecuadamente a los consumidores los riesgos a los cuales se someten al consumir este tipo de productos, sobre todo si se trata de productos alimenticios o que en general- puedan afectar la salud (v.gr. medicinas vencidas).

Se afirma que el consumidor es consciente de la relación entre calidad y precio, y que en alguna medida el bajo precio de los productos que adquiere le informa de la baja calidad de los mismos y de los riesgos que "conscientemente" decide asumir ${ }^{29}$. Discrepamos con esta afirmación.

28 Así por ejemplo podría estar consumiendo cotidianamente carne en estado de descomposición o hamburguesas de papel sin saberlo, sin embargo si de hecho conociese la procedencia y sobre todo las consecuencias que esos productos le pueden generar a la salud, optaría, al ser racional, es decir al tener alternativas de consumo, por no consumirlos.

29 Alfredo Bullard G. Op. cit. p. 107. 
Para nosotros información y "conciencia del riesgo" son conceptos proporcionales, es decir la internalización del riesgo requiere información sobre su existencia. Por ello, y no por otra razón, la mayoría de las críticas al tabaco se dirigen a los fumadores, pues hoy en día es improbable que alguien fume sin ser consciente de los riesgos que su actividad genera a su salud como a la de terceros (v. gr. la posibilidad de contraer cáncer) y ello no sólo porque el producto mismo lo advierta, sino sobre todo por la cantidad de información que existe al respecto (estadística, estudios especializados, información televisiva, etc.).

Distinta sería la situación si dicha información no existiera, pues casi podríamos asegurar que las críticas se dirigirán a los productores por ocultar la información necesaria y relevante para conservar nuestra integridad física, o por lo menos para ser conscientes que la podemos afectar.

El bajo precio de un par de zapatos, de una medicina o de una hamburguesa "al paso" realmente no nos dice mucho, nos dice qué no esperar del producto como por ejemplo, que mis zapatos duren toda la vida, que la medida sea $100 \%$ eficaz o que la hamburguesa tenga realmente buen sabor; sin embargo, la información relevante que deberían contener dichos productos no nos la da el precio (v.gr. que mis zapatos se deshacen en la lluvia porque su suela es de cartón, que la medicina es barata porque está vencida o que mi hamburguesa no es tan agradable por que está hecha de papel y sazonada con "ladrillo" en polvo). Es esta información la que no existe, la que se reclama y la que causa la mayor cantidad de externalidades a los consumidores, sobre todo daños a la salud.

Una segunda razón es el "costo de la experiencia". Se afirma que en un mercado competitivo la información que no brinda el mercado, la provee la experiencia, dado que errar es humano y que no se concibe una economía de mercado en la que no exista un constante aprendizaje ${ }^{30}$.

Creemos que esta afirmación es válida, pero no absoluta en la medida que no toma en consideración las consecuencias de aplicar este razonamiento al mundo de los "productos basura".

Hasta qué punto podemos obligar a las personas a que experimenten con su salud, con su vida ${ }^{31}$; hasta qué punto podemos permitir que

30 Ibid. p. 105.

31 En ese sentido. E.Elías Laroza. “ $i$ Lo barato sale caro: mata y no engorda $i$ La inacción 
los consumidores (sobre todo los pobres y pobres extremos) asuman el sobrecosto de esta experimentación y de la asignación ineficiente de sus recursos.

Definitivamente, allí reside parte del problema. Una cosa es hablar de productos que dado su nivel de calidad afectan -de existir un error en la elección- solamente niveles de satisfacción del consumidor, y algo muy diferente es referirse a productos que dada su función(satisfacción de necesidades básicas) y calidad (pésima) afectan -de mediar error en la elección- la integridad física de sus consumidores.

La tercera razón para corregir la asimetría de información es que ésta no siempre proviene del alto costo de incorporarla, sino que en la mayoría de casos proviene de la conducta dolosa de los proveedores. En realidad la información cuesta y es razonable que el precio de un producto se incremente conforme aumenta la cantidad de información que contiene; por ello es distinta la conducta de quien no incorpora información para reducir sus costos y ofrecer un producto más barato, a la conducta de quien no incorpora información (ni siquiera la relevante) porque sabe que si lo hace, su producto no podrá ser comercializado ${ }^{32}$.

Como es posible comprobar, la conducta inescrupulosa de los proveedores de "productos basura" -en cuanto al ocultamiento de información relevante- no se origina en una decisión de mercado sino en la voluntad y conciencia (dolo) de dañar la salud o integridad de los consumidores a un bajo costo (para los productores) ${ }^{33}$.

En conclusión, no corregir la asimerría de información que existe en el mercado de producto basura (a pesar de las razones expuestas), so pretexto de mantener productos baratos para consumidores pobres, es fomentar la afectación de bienes jurídicos que el propio Estado ha

del INDECOPI ante los productos basura, los cañazos y los yonques". En Ius et Veritas No $^{\circ}$ 13 p. 57.

32 Así por ejemplo quien fabrica embutidos con carne descompuesta o de algún animal no apto para el consumo humano, difícilmente podría pensar que incorporando dicha información al envase de sus productos, estos sean más comperitivos, por el contrario sabe que si lo hace no sólo no se le compran sino que le cierran el local, lo multan, eventualmente tendría que indemnizar a las víctimas y hasta podría "ganarse" 6 años de pena privariva de la libertad (Cod. Penal 91. Delitos contra la Salud Pública. Art. 288).

33 Ello explica en última instancia por qué el mercado no puede corregir la información asimétrica por si mismo y por qué no es capaz de generar los incentivos adecuados para que los productores incorporen calidad y seguridad a sus productos. 
decidido proteger; consentir la generación de externalidades (dados los "costos de la experiencia") que terminan afectando a las personas de más bajos ingresos; es inducir una asignación ineficiente de los recursos.

\section{Las nuevas fronteras de la defensa del consumidor.}

\subsection{Las fronteras actuales.}

Analizada la problemática del consumo en un país con altos niveles de pobreza como el Perú, es inmediata la pregunta ¿cuál es la actitud del Estado (en principio) en defensa del consumidor?

La respuesta es desconcertante, paradójica y formalmente asume como suyo todo cuanto venimos planteando como posible solución a la realidad del "consumo basura" en nuestro país, propuesta que podría resumirse en el control de calidad e información de los productos denominados basura, para brindar de esta forma, seguridad a un consumidor que dada su situación de pobreza no posee en los hechos, capacidad de elección relevante.

Así, el Decreto Legislativo $716^{\circ}$, Ley de Protección al Consumidor, consagra como derechos del consumidor, el de ser protegido eficazmente contra los productos que representen un riesgo o peligro para la salud o la seguridad física (Art. $5^{\circ}$ inc. a); y el de recibir de los proveedores toda la información necesaria para poder tomar una decisión adecuada (Art. $5^{\circ}$ inc. b); señala asimismo como obligación de los proveedores la de cumplir con las normas de seguridad y calidad de los productos (Art. 7o); además cuando esta norma define el concepto de producto, no diferencia entre productos de buena, mala o pésima calidad, por lo que en principio la intervención del mercado en defensa del consumidor no sólo estaría justificada, sino que al mismo tiempo normada.

Si a esto sumamos que el Decreto Ley No 25868 establece que el INDECOPI es el organismo encargado de la aplicación de las normas legales destinadas a proteger a los consumidores (Art. $2^{\circ}$ inc.a); que es el encargado de la aplicación de las normas legales destinadas a proteger la calidad de los productos (Art. $2^{\circ}$ ) y que su actuación no depende de la existencia de alguna denuncia, sino que tiene plenas facultades 
para investigar y actuar de oficio (Art. $47^{\circ}$ inc.b y c), facultades ratificadas y ampliadas por el Decreto Legislativo $807^{\circ}$; no se entiende porqué no se ven los resultados. $\mathrm{E}$ allí lo desconcertante.

Tal vez sea así porque INDECOPI ha optado por no asumir estas funciones y dedicar su esfuerzo a la fiscalización del sector formal de la economía y dejar de lado el sector informal ${ }^{14}$, que es básicamente el medio en el cual se comercializan los productos basura; tal vez sea que dada su actual cantidad de funciones y carga de trabajo no puede avocarse a este nuevo ámbito de protección al consumidor sin descuidar, a la vez, otros no menos importantes.

Sin embargo creer que el INDECOPI es la única institución llamada a defender los intereses de los consumidores, es asumir un concepto limitado de lo que la defensa del consumidor significa, con ello no justificamos su inacción, pero en última instancia la defensa del consumidor es tarea de todos, incluso de los propios consumidores (como se verá más adelante).

Creemos sí que la inacción de los hoy encargados oficiales de la defensa del consumidor frente a la problemática de los productos basura (INDECOPI), se explica por el enfoque limitado que se tiene del problema.

En la actualidad esta temática no ha consentido un análisis desde la perspectiva de la pobreza, por el contrario ésta se asume y lo único que se pretende resolver son los problemas de información asimétrica ${ }^{35}$.

Esta es la actual frontera y límite de la defensa del consumidor en el Perú, razón por la cual la problemática del consumo y de los productos basura existe y subsiste.

\section{2. ¿Por qué ampliar las fronteras?}

Como hemos analizado a lo largo de este trabajo, la temática del consumidor pobre no es sólo un problema de información asimétrica (esta se da pero no es el único), así como tampoco la problemática de la información es un tema que se agote en el ámbito del consumo de pro-

34 Como sugiere Elías Laroza, op.cit. p. 60.

35 Como sostiene Alfredo Bullard "La Problemática de la prorección del consumidor se centra en el tema de la información asimétrica entre el proveedor y el consumidor". A. Bullard Gonzáles, Op. cit. p. 110. 
ductos basura. Por ello si el enfoque de la defensa del consumidor se mantiene con miras a corregir solamente la asimetría informática, corre el riesgo de dejar fuera problemas tan o más trascendentes que el de la información (tanto social como económicamente, como la irracionalidad del consumidor o la generación exagerada de externalidades, problemas que reclaman una pronta solución que paradójicamente, el mercado no puede darla. Un diagrama esquematiza mejor esta situación:

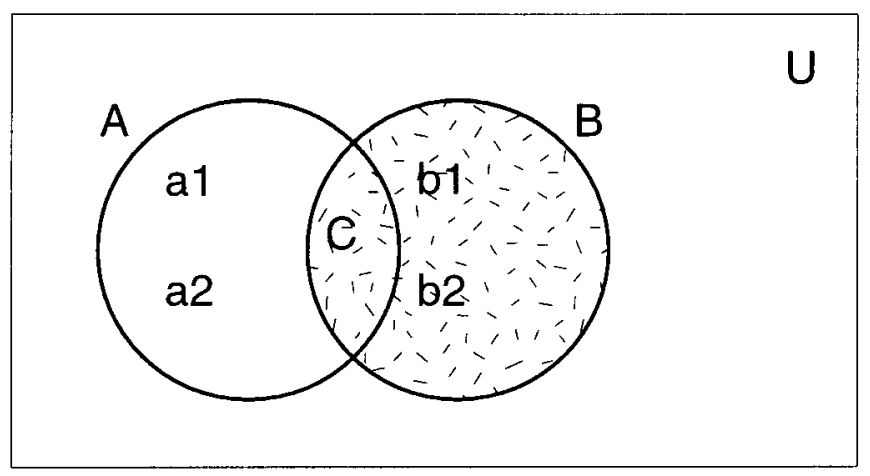

Donde:

U es nuestra sociedad de consumo.

A es la problemática de consumo generada por la pobreza.

$\mathrm{B}$ es la problemática generada por desinformación.

C son problemas generados por pobreza y desinformación.

A1 y A2 son problemas de consumo generados por pobreza y no por desinformación ( $\mathrm{vg}$ irracionalidad del consumidor $\mathrm{y}$ cierto tipo de externalidades).

b1 y b2 son problemas de consumo generados sólo por información asimétrica (vg riesgos inconscientes).

El ámbito actual de la defensa del consumidor.

Como se observa, hoy en día la defensa del consumidor cubre solo el conjunto $\mathrm{B}$, cuando debería cubrir el conjunto AUB.

Asumiendo el riesgo de ser reiterativos, recordemos que la pobreza es la situación del consumidor; lo oprime y a la vez elimina sus posibilidades de elección, convirtiéndolo en un individuo irracional (en el plano del consumo), todo lo cual lejos de aportar a la solidificación de 
los mercados, los deprime al deprimir la demanda, más aún cuando en esta compleja situación, irrumpen los "productos basura" sin control alguno para -aprovechando la irracionalidad del consumidor- generar no sólo pingües ganancias para algunos pocos proveedores sino sobre todo, elevadísimos costos para la gran mayoría de consumidores.

Razones por demás suficientes para enfocar la problemática del "consumo basura" desde la perspectiva de la pobreza y así ampliar las fronteras de la defensa del consumidor. Un enfoque desde la pobreza como hemos pretendido demostrar- es útil y vigente en la medida que aporta nuevos criterios de análisis y cuestiona otros tantos que se habían dado por válidos.

\subsection{Control y pobreza.}

La defensa del consumidor bajo esta orientación más realista puede contribuir en mucho, para ello la palabra clave es control. El control de la calidad y de información de los productos basura parece ser la solución más adecuada. Como vimos en el capítulo anterior, el mercado no puede crear los incentivos necesarios para que los proveedores incorporen calidad, seguridad e información a sus productos, es necesario pues que el Derecho cree estos incentivos para promover una asignación más eficiente de los recursos que se traduzca en un mayor bienestar de los consumidores.

Por ello el control de calidad debe traducirse en un esfuerzo por reducir (internalizar) las externalidades que los productos de pésima calidad generan tanto a la economía como a la salud de sus consumidores.

Este control ayudará a mitigar las consecuencias de la irracionalidad del consumidor, problema generado también por la pobreza pero que la defensa del consumidor no puede corregir directamente, pues la solución responde más a una adecuada política económica que a un efectivo control de calidad de los productos. En este sentido de lo que se trata es que el control logre que aquellos consumidores sin posibilidades de elección, consuman en cada producto un mínimo de calidad (seguridad) y no les genere un perjuicio adicional (externalidad) que de por sí implica consumir productos de mala calidad.

En cuanto al control de información -corrección de información asimétrica- es claro que desde un enfoque tradicional de la defensa del 
consumidor, este problema puede encontrar solución. Sin embargo, es necesario reincidir en él -y un análisis desde la pobreza lo logra- porque hasta ahora ha permanecido olvidado.

Ahora bien, es evidente que el control puede aportar no sólo en la internalización de externalidades, sino también en la prevención de las mismas. Asimismo, el control puede devenir eventualmente en prohibición de determinados productos, sin embargo si no queremos terminar prohibiendo a diestra y siniestra productos basura ni fomentar una sobre protección del consumidor (porque ello distorsionaría aún más las imperfecciones del mercado que queremos corregir), es necesario asumir criterios claros de actuación.

\subsection{Criterios de actuación}

A continuación mencionaremos dos criterios que han sido reiteradamente citados en este ensayo, criterios que se deberían tomar en cuenta en toda política de defensa del consumidor, si quiere evitar un uso ineficiente y eventualmente abusivo de sus prerrogativas.

\section{Primer criterio; necesidades básicas o primarias.}

Como sabemos, los productos basura pueden cubrir una serie de necesidades de los consumidores, desde alimentación hasta transporte. Sin embargo, la gran mayoría de críticas contra los productos basura, se dan en la medida que afectan necesidades básicas, es decir requerimientos inmediatos para mantener la vida ${ }^{36}$ (vg. las medicinas y alimentos), y ello porque las externalidades que generan dañan directamente la salud de los consumidores, siendo éste un costo realmente elevado para quienes se encuentran viviendo en situación de pobreza y pobreza extrema.

Creemos que ello justifica la distinción. La defensa del consumidor debe controlar sobre todo la calidad de aquellos productos basura que

36 Amalia Cuba citando a Georgescu-Roegen, distinguen tres tipos de necesidades: Necesidades Primarias: requerimientos mínimos para mantener la vida.

Necesidades Convencionales: referidas al nivel cultural. Son impuestas por el medio social. Necesidades Puramente Personales: Lujos.

Amalia Cuba Salerno. Op. cit. P. 10. 
se destinan a satisfacer necesidades básicas o primarias de los consumidores, y en cuanto a los productos que cubren otro tipo de necesidades ya convencionales ya puramente personales (suntuarias), el control debe orientarse solamente a corregir la asimetría de información que exista, más no la calidad, salvo en lo que respecta a requerimientos mínimos de seguridad, que deviene en el segundo criterio de análisis.

\section{Segundo Criterio: La seguridad.}

La "seguridad" no es sólo un criterio de análisis, es también un derecho reconocido al consumidor, de allí su importancia ${ }^{37}$.

Nuestro primer criterio nos permitió deslindar claramente el campo de actuación de la defensa del consumidor en cuanto a control de calidad se refiere, enfocándolo directamente a aquellos productos (basura) destinados a satisfacer necesidades primarias. No obstante ello es insuficiente.

En primer lugar dentro de estos productos existen diversas calidades y grados de afectación a la salud de los consumidores. Pretender lograr un control de calidad total puede ser tan ineficiente como ilógico dado que lo que se busca no es la eliminación de los productos basura (en la medida que les obliguemos a ser productos de alta calidad), sino que estos internalicen gran parte de las externalidades que producen.

En segundo lugar que un bien o producto no esté dirigido a satisfacer necesidades básicas, no quiere decir que deje de afectar la salud o integridad de los consumidores.

Por estas razones se justifica el criterio de seguridad, que nos da la pauta para controlar la calidad de productos basura que satisfagan tanto necesidades primarias como de otro tipo.

A partir de los dos criterios mencionados se puede diseñar un cuadro de doble entrada que grafique los distintos ámbitos de actuación de la defensa del consumidor frente al universo de producto del mercado, tanto desde un enfoque tradicional (que solo incorpora el criterio de información) como desde un enfoque que añade la perspectiva de la pobreza ( $\mathrm{y}$ los criterios de necesidad primaria y seguridad).

37 Ver Supra 28. 


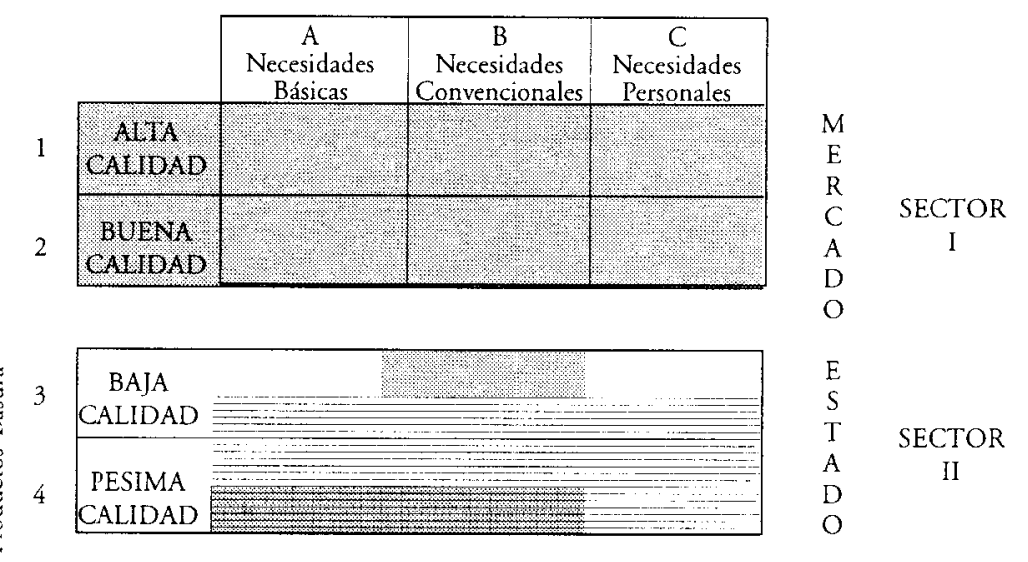

Estándar de seguridad de los productos

Ámbito de control de información

Ámbito que incorpora el control de calidad

Nivel de prohibición de productos

La división en sector I y sector II no es caprichosa, demarca en primer lugar las fronteras de la defensa del consumidor desde un enfoque puramente "informativo" (I) y la medida en que éstas se ven ampliadas desde una perspectiva que incorpora la pobreza como criterio de análisis (II). Como se aprecia, es a partir del nuevo enfoque que las probabilidades de protección incorporan tanto a productos de baja como de pésima calidad, es decir a los productos basura.

Otro aspecto importante son los binomios mercado-sector I y Estado-sector II, ello quiere decir que en el universo de productos de buena y alta calidad, el mercado es autosuficiente para crear los incentivos necesarios que incorporen calidad y seguridad a los productos. De allí que la defensa del consumidor se haya limitado a corregir solamente problemas de información asimétrica. En cambio, en el sector II al ser el mercado insuficiente para crear estos incentivos, se hace necesaria la intervención estatal para controlar calidad y seguridad.

Ahora bien, desde el sector II se puede apreciar la contribución de los criterios de "necesidad básica" y seguridad. El primero nos obliga a 
hacer un control de calidad, en principio, de todos los productos de la columna A, inclusive de los que se encuentren en el sector A1 y A2. Sin embargo, dado que en ellos el mercado puede controlar la calidad y seguridad, la defensa del consumidor debe mantenerse al margen. El segundo criterio amplia este control a la columna B pero solo en lo correspondiente a la calidad mínima que deben brindar los productos basura (stándard de seguridad), para evitar afectaciones a la salud e integridad física de los consumidores. Todo control adicional sólo podrá ser de información (vg. sector B3 parte superior) ${ }^{38}$.

El segundo criterio sirve además para diferenciar cuándo un producto, independientemente del tipo de necesidad que satisfaga, debe ser prohibido. Como se observa en el cuadro, en la medida que existan productos cuya calidad sea inferior a los requerimientos de seguridad, deben prohibirse (vg. las medicinas vencidas).

Finalmente, si el sector $\mathrm{C} 3$ y C4 se mantienen en blanco, es porque no imaginamos la existencia de productos basura que satisfagan lujos, ello más bien es un problema de "productos bamba" ajeno a nuestro análisis. En todo caso de existir estos, les sería aplicable el segundo criterio (seguridad).

Como se observa el control de calidad de los productos no tiene por qué ser dramatizado, ya que éste viene delimitado por criterios claros de actuación, de allí que se aplique sólo a productos de baja y pésima calidad (productos basura) que satisfagan necesidades básicas o primarias de los consumidores, y también a aquellos que abran otro tipo de necesidades pero sólo en lo referente a requerimientos mínimos de seguridad.

38 Un ejemplo nos aclara el panorama. Imaginemos que compramos una bicicleta marca Rayo, definitivamente no se trata de un bien que satisfaga una necesidad básica o primaria, por lo que por allí no vendría dado el control de calidad. Sin embargo al ser un bien que cubre otro tipo de necesidad, de acuerdo al segundo criterio, debe cumplir con requisitos mínimos de seguridad (vg. llantas que no salgan volando en el primer bache, un timón que no se quede en las manos en la primera curva cerrada).

La bicicleta podrá ser de pésima calidad y tal vez no me dure ni 2 meses porque las llantas se desinflan a cada instante, la pintura se sale totalmente, los cromados se oxidan...etc., pero en lo que no deberá fallar es en el mínimo de calidad que la haga segura en la función que realiza. Todo control adicional sólo podrá ser de información, por ello la defensa del consumidor no deberá preocuparse por el tipo de pintura o cromado de la bicicleta o la presión de sus llantas, sin embargo si deberá preocuparse porque los proveedores informen de ello a los consumidores para que no sólo tomen una decisión adecuada sino sobre todo para que sean conscientes de los riesgos del producto y de aquellos otros que deciden asumir. 
Ciertamente se puede criticar que referirnos a "niveles de calidad", "estándares" de seguridad, y necesidades básicas es muy ambiguo. Incluso preguntamos ¡cuándo un producto es de buena calidad, cuándo es inseguro, qué es una necesidad básica?

Creemos que la objeción sería válida, pero esta "relativa" impresión de los conceptos es preferible a la total indefinición que acarrearía indefensión para el consumidor o abusos contra el proveedor. Aludimos a "relativa" imprecisión porque los conceptos cuestionables pueden ser mucho más precisos, por suerte ello no depende del Derecho sino de otras ramas del conocimiento. Así, calidad y seguridad (desde la administración) pueden definirse en función a estándares internacionales, lo cual aporta mucho en precisión. De igual forma, "necesidad primaria" es un concepto que maneja hace mucho la economía y que viene definido a la elasticidad de la demanda del producto.

\subsection{Defensa del consumidor en sentido amplio}

Como habíamos mencionado en otro momento, una visión amplia de la defensa del consumidor nos obliga a ver más allá de INDECOPI y a reparar en las instituciones cuyo objetivo puede ser este pero que tal vez habíamos perdido de vista.

Así, en la medida que la defensa del consumidor sea asumida desde todos estos campos, los resultados comenzarán a verse y la problemática del consumo y de los productos basura dejará de ser tal. A manera de repaso, tenemos:

\section{Defensa del Consumidor:}

- Colectiva Autónoma: Asociaciones de Consumidores

- Heterónoma:

- Directa: INDECOPI (normas sobre Defensa del consumidor).

Resp. Civil (contractual y extracontractual)

Resp. Penal (delitos contra la Salud Pública)

Municipalidad (otorgamiento de licencias y control sanitario).

- Indirecta: Política Económica Estatal

Empresa Privada 
La enumeración no es taxativa pero deja ver que los instrumentos para asumir la defensa del consumidor existen, lo único que requieren es afinar su uso desde un enfoque que incorpore la perspectiva de la pobreza. Sin embargo, el gran impulso en esta política debe darla el Estado quien está obligado a asumir el rol de Promotor que le corresponde, entre otros aspectos.

\subsection{Reflexión final}

"La iniciativa privada es libre. Se ejerce en una economía. Social de mercado. Bajo este régimen, el Estado orienta el desarrollo del país (...)" Art. 58 Constitución Política 1993.

"El Estado defiende el interés de los consumidores y usuarios. Para tal efecto garantiza el derecho a la información sobre los bienes y servicios que se encuentran a su disposición en el mercado. Asimismo vela, en particular, por la salud y la seguridad de la población”. Art. 65 Constitución Política 1993.

Si las normas tuvieran la magia de transformar los problemas en bellas realidades, no estaríamos preocupados por el desfase existente entre el Derecho y la Realidad. Lamentablemente ello no es así.

Pero algo es cierto, las normas serán mágicas y el Derecho responderá a la realidad en la medida que sus postulados dejen de ser líricas declaraciones. Cualquier observador ajeno a nuestra realidad pero consciente de la misma, se sentiría realmente emocionado al leer las frases de los artículos constitucionales citados. Sin embargo, vale preguntarse hasta qué punto estas normas son capaces de convencer a la mitad del país que viven en la pobreza, de que las cosas son como ellas las declaran.

Nadie puede negar que el problema está más allá de la Constitución, ella es sólo una carta fundamental cuya virtud es establecer los pilares en los que nuestra sociedad debe descansar. En esa medida los artículos $58^{\circ}$ y $65^{\circ}$ son absolutamente necesarios para crear una adecuada política de defensa del consumidor, pero no suficientes.

Lo que se reclama es que el Estado (en tanto defensor de los intereses de los consumidores), termine de convencerse que nuestra economía es también Social y no sólo de mercado; que ello implica una 
actitud decidida que busque corregir las desigualdades pre-existentes que cuestionan constantemente los presupuestos del mercado.

De lo que se trata es de crear mercados capaces de resistir el juego libre de la economía, no de liberalizarlos porque si a sabiendas que no estamos preparados para ello, y bajo el riesgo de incrementar la desigualdad que queremos corregir.

Desde este punto de vista no existe prejuicios ni perjuicios en promover una auténtica defensa del consumidor que en una realidad pobre como la nuestra requiere del Estado (aunque no solo de él) para alcanzar sus fines. Lo prejuicioso y perjudicial es cuestionar a priori la participación del Estado en la economía y dar por cierto que toda intervención suya tiende a ser arbitraria e ineficiente.

Todo Estado moderno aspira a que sus mercados funcionen bien, sean eficientes y contribuyan al bienestar social. Sería un contrasentido que preocupándose tanto los estados por establecer reglas de juegos claras para la economía, se dediquen luego a desconocerlas, y aunque al parecer nuestra historia ha sido un reencuentro infinito de estas situaciones (y de allí la desconfianza), es necesario advertir que ni siquiera el mercado puede evitar que las reglas de juego se desconozcan, si algún gobernante "innovador" decide hacerlo.

Si en nuestros tiempos es momento de creer en el mercado, debemos también aprender a creer en el Estado, no como un economista brillante, sino como el promotor de una economía brillante. Ello lo obliga entre otras cosas a corregir las imperfecciones del mercado en la medida de que éste no logre hacerlo por sí mismo.

En el tema particular que hemos tratado las cosas no son diferentes. La defensa del consumidor es un campo en el cual la tutela estatal no puede retroceder y el mercado de los productos de baja y pésima calidad es uno que no puede liberalizar, sin tomar en cuenta el aspecto social (pobreza). No sólo por un mandato constitucional que limite al Estado en este sentido, sino además porque una real economía social de mercado presupone la existencia de mercados, y ellos aún están en formación en el Perú.

Algún día llegará en que los mercados sean tan eficientes y perfectos que podrán dar solución a cuanto problema social se presente, sin requerir para nada de la "ayuda" del Estado. A la vez, si éstos no quieren desaparecer tendrán que volverse igualmente eficientes. Si ello no es así, quizás las sociedades decidan instituirse en función a un orden 
económico ya no estatal, pero hasta entonces (en nuestro país todavía falta mucho para ello) tanto el Estado como el Derecho y la defensa del consumidor tienen y tendrán mucho qué hacer. 